\title{
Morphology of the Pelvis and Hind Limb of the Red Panda (Ailurus fulgens) Evidenced by Gross Osteology, Radiography and Computed Tomography
}

\author{
M. Makungu ${ }^{1,2 \star}$, W. M. du Plessis ${ }^{3}$, H. B. Groenewald ${ }^{1}$, M. Barrows ${ }^{4}$ and K. N. Koeppel ${ }^{5}$ \\ ${ }^{1}$ Department of Anatomy and Physiology, Faculty of Veterinary Science, University of Pretoria, Private \\ Bag X04, Onderstepoort 0110, South Africa; \\ ${ }^{2}$ Department of Veterinary Surgery and Theriogenology, Faculty of Veterinary Medicine, Sokoine \\ University of Agriculture, P. O. Box 3020, Morogoro, Tanzania; \\ ${ }^{3}$ Ross University School of Veterinary Medicine, P. O Box 334, Basseterre, St. Kitts, West Indies; \\ ${ }^{4}$ Bristol Zoo Gardens, Clifton, Bristol BS8 3HA, UK; \\ ${ }^{5}$ Johannesburg Zoo, Private Bag X13, Parkview, Johannesburg 2122, South Africa
}

\section{${ }^{*}$ Correspondence:}

Tel.: +27 12529 8246;

fax: +27 125298320 ;

e-mail: modesta_makungu@yahoo.com

With 9 figures and 3 tables

\section{Summary}

The red panda (Ailurus fulgens) is a quadrupedal arboreal animal primarily distributed in the Himalayas and southern China. It is a species commonly kept in zoological collections. This study was carried out to describe the morphology of the pelvis and hind 
limb of the red panda evidenced by gross osteology, radiography and computed tomography (CT) as a reference for clinical use and identification of skeletons. Radiography of the pelvis and right hind limb was performed in nine and seven animals, respectively. Radiographic findings were correlated with bone specimens from three adult animals. Computed tomography of the torso and hind limb was performed in one animal. The pelvic bone had a wide ventromedial surface of the ilium. The trochlea of the femur was wide and shallow. The patella was similar to that seen in feline species. The medial fabella was not seen radiographically in any animal. The cochlea grooves of the tibia were shallow with a poorly defined intermediate ridge. The trochlea of the talus was shallow and presented with an almost flattened medial ridge. The tarsal sesamoid bone was always present. The lateral process of the base of the fifth metatarsal (MT) bone was directed laterally. The MT bones were widely spaced. The morphology of the pelvis and hind limb of the red panda indicated flexibility of the pelvis and hind limb joints as an adaptation to an arboreal quadrupedal lifestyle.

\section{Introduction}

The red panda (Ailurus fulgens) is a quadrupedal arboreal animal primarily distributed in the Himalayas and southern China (Fisher et al., 2009). It is classified by the International Union for Conservation of Nature and Natural Resources (IUCN) as a vulnerable species (IUCN, 2014). The red panda is closely related to mustelids, procyonids and skunks (Groves, 2011).

Wild pandas weigh between 4.0 to $5.0 \mathrm{~kg}$ (Preece, 2011). In captivity, the weight of adult males and females ranges from 3.7 to $6.2 \mathrm{~kg}$ (mean: $5.0 \mathrm{~kg}$ ) and 4.2 to $6.0 \mathrm{~kg}$ 
(mean: $4.9 \mathrm{~kg}$ ), respectively (Roberts and Gittleman, 1984). The head and body length ranges from 560 to $625 \mathrm{~mm}$. The tail length ranges from 370 to $472 \mathrm{~mm}$ (Roberts and Gittleman, 1984). The red panda lives in forests with bamboo understory at an altitude ranging from 1500 to 4800 metres (Srivastav et al., 2009). Trees provide sites for resting, sleeping, nesting and escaping from predators (Roberts and Gittleman, 1984; Srivastav et al., 2009; Eriksson et al., 2010). Apart from trees they also prefer other elevated surfaces such as rocks (Roberts and Gittleman, 1984; Pradhan et al., 2001). Although the red panda spends most of its time in trees, it forages primarily on the ground (Roberts and Gittleman, 1984). Its diet is largely vegetarian, consisting primarily of bamboo leaves [80-90\%] (Wei and Zhang, 2009). The average lifespan of the red panda in the wild is 8 to 10 years, whereas in captivity it is 13.4 years (Heath and Platnick, 2008).

Diseases involving the musculoskeletal system such as avascular necrosis of the femoral head (Delclaux et al., 2002), hyperostotic bone disease (Lynch et al., 2002), osteoarthrosis (Philippa and Ramsay, 2011; Preece, 2011) and neoplasia such as squamous cell carcinoma (Preece, 2011) have been reported in the red panda. Although radiography has been used as the first diagnostic imaging modality for musculoskeletal disease in the red panda (Delclaux et al., 2002; Lynch et al., 2002), computed tomography (CT) is an excellent imaging modality to evaluate bone (Ohlerth and Scharf, 2007). Compared to radiography, CT provides cross-sectional images without superimposition and has superior tissue contrast. Therefore, early bone changes can be detected (Ohlerth and Scharf, 2007). Further, post-processing of images (multiplanar and three-dimensional reconstructions) is possible with the CT (Kinns et al., 2011). 
Normal osteology, radiographic and CT anatomy of the pelvis and hind limb are well documented in companion animals (Nickel et al., 1986; Samii et al., 2011; Thrall and Robertson, 2011) and provide a reference for identification of skeletons and diagnosis of musculoskeletal diseases. Several authors have also documented the normal osteology, radiographic and CT anatomy of the pelvis and hind limb in wildlife species (Taylor, 1976; Silverman and Tell, 2005; de Araújo et al., 2013).

There are few reports on the normal gross osteology of the pelvis and hind limb of the red panda (Roberts and Gittleman, 1984; Fisher et al., 2008; Fisher, 2011) and to the authors' knowledge, the normal radiographic and CT anatomy of the pelvis and hind limb has not been reported in this species. Interestingly, the red panda has been reported to descend head first by gripping the tree trunk medially with the feet (Roberts and Gittleman, 1984), indicating how well the hind limb is adapted for arboreal quadrupedal locomotion. The objective of this study was to document the normal gross osteology, radiographic and CT anatomy and range of variation in the pelvis and hind limb of the red panda with the aim of helping identify lesions successfully.

\section{Materials and Methods}

\section{Animals}

Nine captive adult red pandas (Ailurus fulgens) from the Johannesburg (JHB) zoo were radiographed in this study (Table 1). Six were males, and three were females (Table 1). The minimum and maximum ages of the animals were 1.4 and 14.3 years, respectively (mean: $6.76 \pm 4.96$ years). The weight of the animals ranged from 3.7 to $5.96 \mathrm{~kg}$ (mean: $4.65 \pm 0.78 \mathrm{~kg}$ ). Of the nine animals, two were euthanased during the course of the 
Table 1. Sample population of red pandas used for gross osteology, radiography and computed tomography $(\mathrm{CT})$

\begin{tabular}{lllll}
\hline Identification & $\begin{array}{l}\text { Age } \\
(\text { years })\end{array}$ & $\begin{array}{l}\text { Weight } \\
(\mathrm{kg})\end{array}$ & Sex & Study \\
& 14.3 & 3.7 & Male & Gross osteology, radiography and CT \\
\hline $3323^{\mathrm{a}}$ & 11.3 & 3.87 & Male & Gross osteology and radiography \\
$4694^{\mathrm{a}}$ & 10.3 & 5.09 & Male & Radiography \\
5230 & 9.6 & 4.65 & Female & Radiography \\
5542 & 2.5 & 4.57 & Male & Radiography \\
8493 & 1.4 & 3.97 & Female & Radiography \\
8680 & 1.4 & 5.6 & Male & Radiography \\
8681 & 2.08 & 4.48 & Female & Radiography \\
8678 & 8 & 5.96 & Male & Radiography \\
6591 & 3.5 & & Male & Gross osteology \\
$2004.71 .2^{\mathrm{b}}$ & & & & \\
\hline
\end{tabular}

${ }^{\mathrm{a} E}$ Euthanized animals

${ }^{\mathrm{b}}$ Bone specimens from the National Museum of Scotland

study and were subjected to gross osteological study (Table 1). Bone specimens of a 3.5-year-old male red panda from the National Museum of Scotland were also used for gross osteological study (Table 1). This study was approved by the Animal Use and Care Committee (AUCC) of the University of Pretoria and Johannesburg Zoo Ethics and Research Committee. 


\section{Radiography}

Radiography of the pelvis and right hind limb was performed in nine and seven animals, respectively, under general anaesthesia using a table top technique. Animals were radiographed during annual health examinations. In eight animals, a EVA-HF525 X-ray machine (Comed Medical System Co. Ltd, Kyunggi, Korea) was used at a source to image distance (SID) of $95 \mathrm{~cm}$. An automatic X-ray film processor model CP-345 (ELK Corporation, Tokyo, Japan) was used. For the pelvis, a kVp range of $42-44$ and 2.5 mAs was used with medium speed screen type film, Fuji medical X-ray films (Fujifilm Corporation, Tokyo, Japan) in combination with RAREX green regular intensifying screens (Okamoto manufacturing Co. LTD, Tokyo, Japan). For the hind limb, $46 \mathrm{kVp}$ and $5 \mathrm{mAs}$ was used with mammography films (UM-MA, Fujifilm Corporation, Tokyo, Japan) in combination with mamoray screens (Fujifilm Europe $\mathrm{GmbH}$, Düesseldorf, Germany). In the remaining animal, radiography was performed using a Siemens Polymat 50 (Siemens, Munich, Germany) X-ray machine at a SID of $100 \mathrm{~cm}$. Images were obtained using a computed radiography (CR) unit (Fuji Axim FCR Capsula XL; Fujifilm Corporation). A kVp range of $48-60$ and $m A s$ range of $4-6$ was used for the pelvis and hind limb, respectively.

\section{Computed tomography}

Computed tomography of the torso and the left hind limb was performed in one animal (Table 1) using a Siemens Emotion Duo CT scanner (Siemens Medical Systems, Forchheim, Germany). For the torso, 2-mm-thick transverse slices were acquired from just cranial to the thoracic inlet to the ischiatic tuberosities at a kV of 130 and $20 \mathrm{mAs}$ using a soft tissue window [window width $(\mathrm{WW})=400$ and window level $(\mathrm{WL})=40$ ] and 
a medium kernel (B41s). For the hind limb, 1-mm-thick transverse slices were acquired from just proximal to the stifle joint to the distal phalanges using a bone window (WW $=$ 1400 and $\mathrm{WL}=300$ ) at a $\mathrm{kV}$ of 130 and $30 \mathrm{mAs}$ with a sharp kernel (B80s). A postprocessing, three-dimensional (3D) volume rendering technique was performed with a grey scale default.

\section{Gross osteology}

Bone specimens from three adult animals (National Museum of Scotland and two euthanased animals from the JHB zoo) were used (Table 1). Photographs of bone specimens from different views were obtained using a digital camera (CANON 5DMARK2, Canon Inc., Tokyo, Japan). A previous report (Fisher et al., 2008) on the myology of the pelvis and hind limb of the red panda was used to locate the sites for origins and insertions of different muscles on bone specimens and their functions.

\section{Bone measurements}

Bone measurements of the pelvis and the right hind limb were performed on the radiographic images. The maximum lengths of bones were measured from the proximal to the distal extremities on the mediolateral (ML) view. For the metatarsal (MT) bones, phalanges and tarsal sesamoid bone, the maximum lengths were measured on the plantarodorsal (PD) view of the pes. The craniocaudal (CrCd) and ML diameters were measured on the ML and PD views, respectively. The CrCd diameters of the long bones were measured at the mid-diaphysis. For the sesamoid bones, the $\mathrm{CrCd}$ and $\mathrm{ML}$ diameters were measured as a maximum distance perpendicular to the length of the bone. The maximum length of the pelvis was measured on the ventrodorsal (VD) view 
between two lines drawn transversely from the most cranial part of the right wing of the ilium and the most caudal part of the right ischium. The transverse diameter was measured on the VD view from one iliopectineal line to the other (O'Brien, 1978). Radiographic measurements were not compensated for magnification.

\section{Statistical analysis}

Data were analysed using Stat $\operatorname{View}^{\odot}$ (SAS Institute Inc., Cary, NC, USA) statistical package. The mean, range and standard deviation (SD) were calculated. Data are expressed as mean $\pm \mathrm{SD}$ and the range.

\section{Results}

\section{Pelvis}

Gross osteology

The ilial wings were broad, and the gluteal surface was directed slightly dorsolaterally (Figs $1 a, b$ and 2a). When viewed from dorsally or ventrally, the ilial wings were almost parallel to each other (Fig. 1a,b). The body of the ilium and the major ischiatic notch were short (Figs 1a,b and 2a). The ventromedial surface of the ilium was wide (Fig. 1b). The origin for the $\mathrm{m}$. rectus femoris presented with a rough surface (Fig. 2a). The pubic bone was L-shaped with a wide cranial and caudal ramus (Figs 1a,b and 2a). The pelvic symphysis was partially fused in two of the three bone specimens (Fig. 1a,b). The obturator foramina were triangular with rounded edges (Fig. 2a). The ischiatic tuberosities were not prominent (Fig. 1a). The ischiatic arch was deep and narrow (Fig. 
1a). The grooves for the tendon of the m. obturatorius internus were prominent (Figs 1a and 2a).
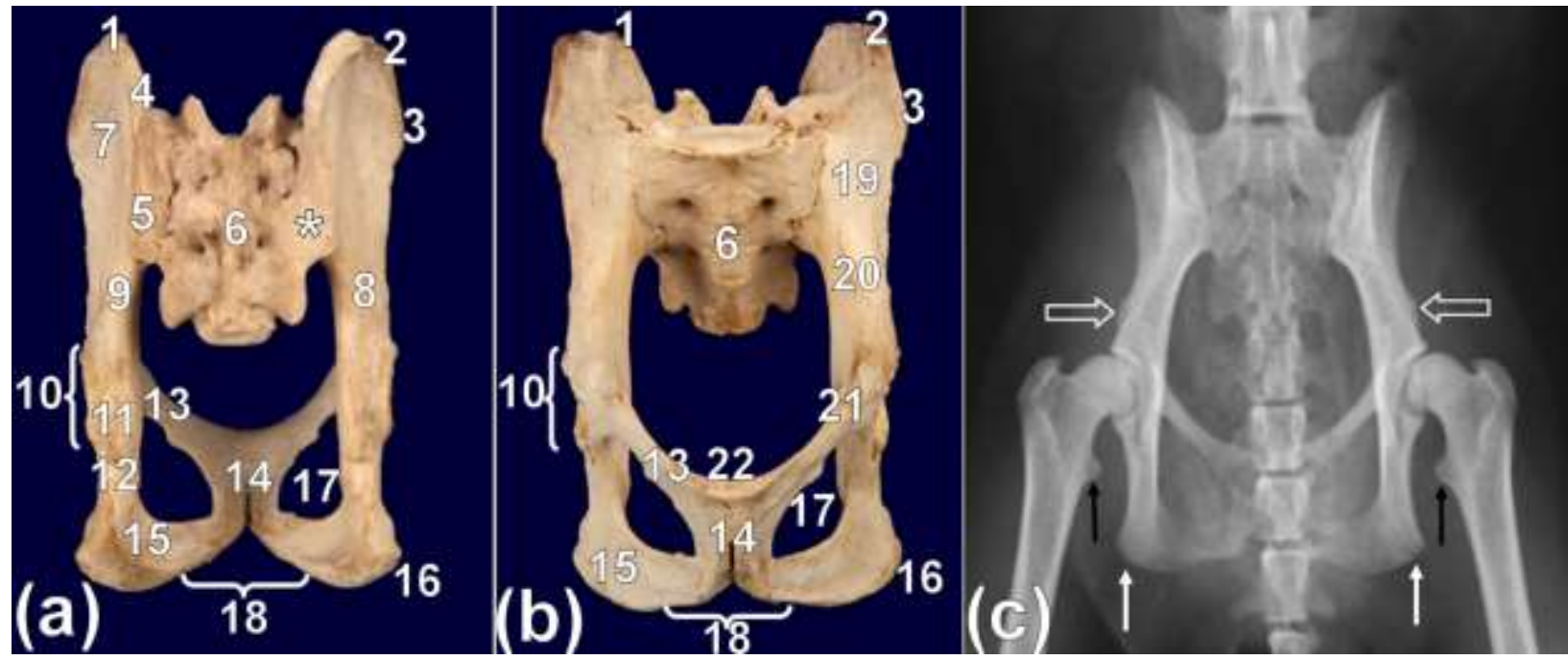

Fig. 1. Dorsal (a) and ventral (b) views of a bone specimen of the pelvis of a 14.3-year-old male red panda. (c) Ventrodorsal radiograph of the pelvis of a 2.1-year-old female red panda. Note the fusion of the dorsal surface of the wing of the sacrum and ilium (asterisk) (a) and the cranial part of the pelvic symphysis (a, b). Note also the wide ventromedial surface of the ilium (b). (c) The ilial wings are wide, and the lateral area for the $\mathrm{m}$. rectus femoris (open white arrows) and ischiatic tuberosities (white arrows) are less prominent. The prominent minor trochanters of the femur are pointed at by black arrows. (1) iliac crest, (2) cranial ventral iliac spine, (3) caudal ventral iliac spine, (4) cranial dorsal iliac spine, (5) caudal dorsal iliac spine, (6) sacrum, (7) gluteal surface, (8) body of ilium, (9) major ischiatic notch, (10) acetabulum, (11) ischiatic spine, (12) groove for the tendon of the m. obturatorius internus, (13) pubic bone, (14) pelvic symphysis, (15) ischium, (16) ischiatic tuberosity, (17) obturator foramen, (18) ischiatic arch, (19) iliac surface, (20) arcuate line, (21) iliopubic eminence, (22) pecten of pubic bone.

\section{Radiography and CT}

On the VD view, the pelvic inlet was fairly rounded and the obturator foramina appeared fusiform (Fig. 1c). The body of the ischium was fairly straight, and the ischiatic tuberosities were not prominent (Fig. 1c). On the right lateral $(R L)$ view, the obturator 


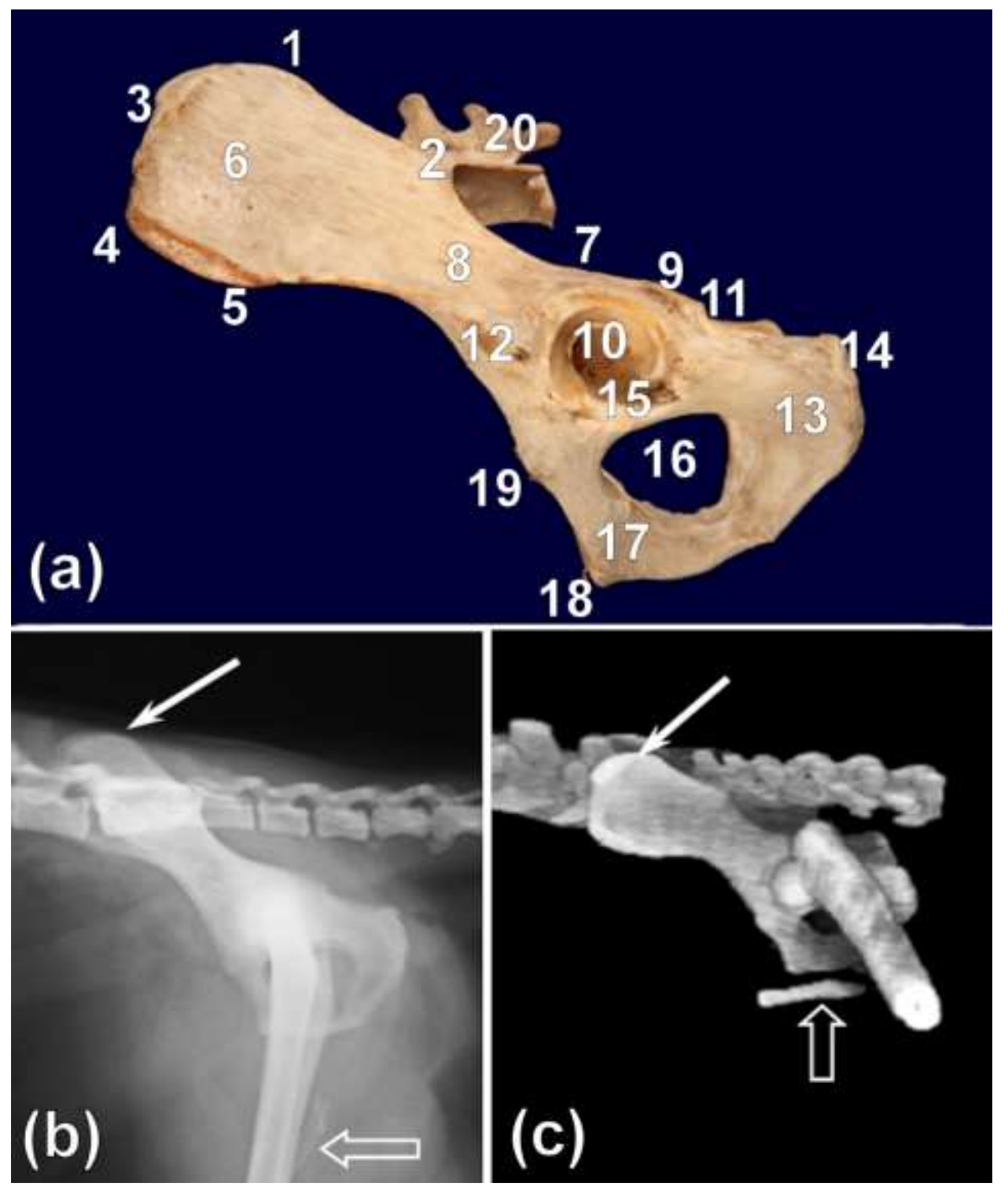

Fig. 2. Lateral view (a) of a bone specimen of the pelvis of a 14.3-year-old male red panda. Right-lateral radiograph (b) of the pelvis of an 8-year-old male red panda. Lateral view (c) of a 3D volume-rendered computed tomography image (using a grey scale default) of the pelvis of a 14.3-year-old male red panda. Note the broad ilial wings (white arrows) and visualization of the os penis (open white arrow). (1) cranial dorsal iliac spine, (2) caudal dorsal iliac spine, (3) iliac crest, (4) cranial ventral iliac spine, (5) caudal ventral iliac spine, (6) gluteal surface, (7) major ischiatic notch, (8) body of the ilium, (9) ischiatic spine, (10) acetabulum, (11) groove for the tendon of the m. obturatorius internus, (12) lateral area for the $\mathrm{m}$. rectus femoris, (13) ischium, (14) ischiatic tuberosity, (15) acetabular notch, (16) obturator foramen, (17) pubic bone, (18) pecten of pubic bone, (19) iliopubic eminence, (20) sacrum. 
foramina appeared triangular with rounded angles (Fig. 2b). The iliac crest was straight, and the pelvic symphysis was short (Fig. 2b,c). A prominent os penis was seen in all male animals (Fig. 2b,c). Radiographic measurements are summarised in Table 2.

Table 2. Radiographic measurements, not compensated for magnification, of the pelvis, long bones and sesamoid bones of the right hind limb in captive red pandas

\begin{tabular}{|c|c|c|c|c|}
\hline Bone & Variables & $\begin{array}{l}\text { Number of } \\
\text { animals }\end{array}$ & $\begin{array}{l}\text { Mean } \pm \text { SD } \\
(\mathrm{cm})\end{array}$ & Range $(\mathrm{cm})$ \\
\hline \multirow[t]{2}{*}{ Pelvis } & Length & 8 & $9.25 \pm 0.21$ & $8.90-9.50$ \\
\hline & T diameter & 8 & $3.45 \pm 0.22$ & $3.20-3.70$ \\
\hline \multirow[t]{2}{*}{ Femur } & Length & 6 & $11.98 \pm 0.37$ & $11.50-12.50$ \\
\hline & CrCd diameter & 6 & $0.80 \pm 0.06$ & $0.70-0.90$ \\
\hline \multirow[t]{2}{*}{ Tibia } & Length & 6 & $11.52 \pm 0.32$ & $11.10-12.00$ \\
\hline & CrCd diameter & 6 & $0.78 \pm 0.42$ & $0.70-0.80$ \\
\hline \multirow[t]{2}{*}{ Fibula } & Length & 6 & $10.42 \pm 0.33$ & $10.00-10.90$ \\
\hline & CrCd diameter & 6 & $0.30 \pm 0.00$ & $0.30-0.30$ \\
\hline \multirow[t]{2}{*}{ Patella } & Length & 7 & $1.19 \pm 0.02$ & $1.15-1.20$ \\
\hline & CrCd diameter & 7 & $0.43 \pm 0.05$ & $0.40-0.50$ \\
\hline \multirow[t]{2}{*}{ Lateral fabella } & Length & 5 & $0.17 \pm 0.07$ & $0.10-0.25$ \\
\hline & CrCd diameter & 5 & $0.26 \pm 0.15$ & $0.10-0.40$ \\
\hline \multirow[t]{2}{*}{ Popliteal sesamoid } & Length & 5 & $0.21 \pm 0.02$ & $0.20-0.25$ \\
\hline & CrCd diameter & 5 & $0.39 \pm 0.02$ & $0.35-0.40$ \\
\hline \multirow[t]{2}{*}{ Tarsal sesamoid } & Length & 5 & $0.70 \pm 0.17$ & $0.60-1.00$ \\
\hline & ML diameter & 5 & $0.29 \pm 0.02$ & $0.25-0.30$ \\
\hline \multirow[t]{2}{*}{ Metatarsal sesamoid } & Length & 4 & $0.31 \pm 0.09$ & $0.20-0.40$ \\
\hline & CrCd diameter & 4 & $0.14 \pm 0.03$ & $0.10-0.15$ \\
\hline
\end{tabular}

T, Transverse 


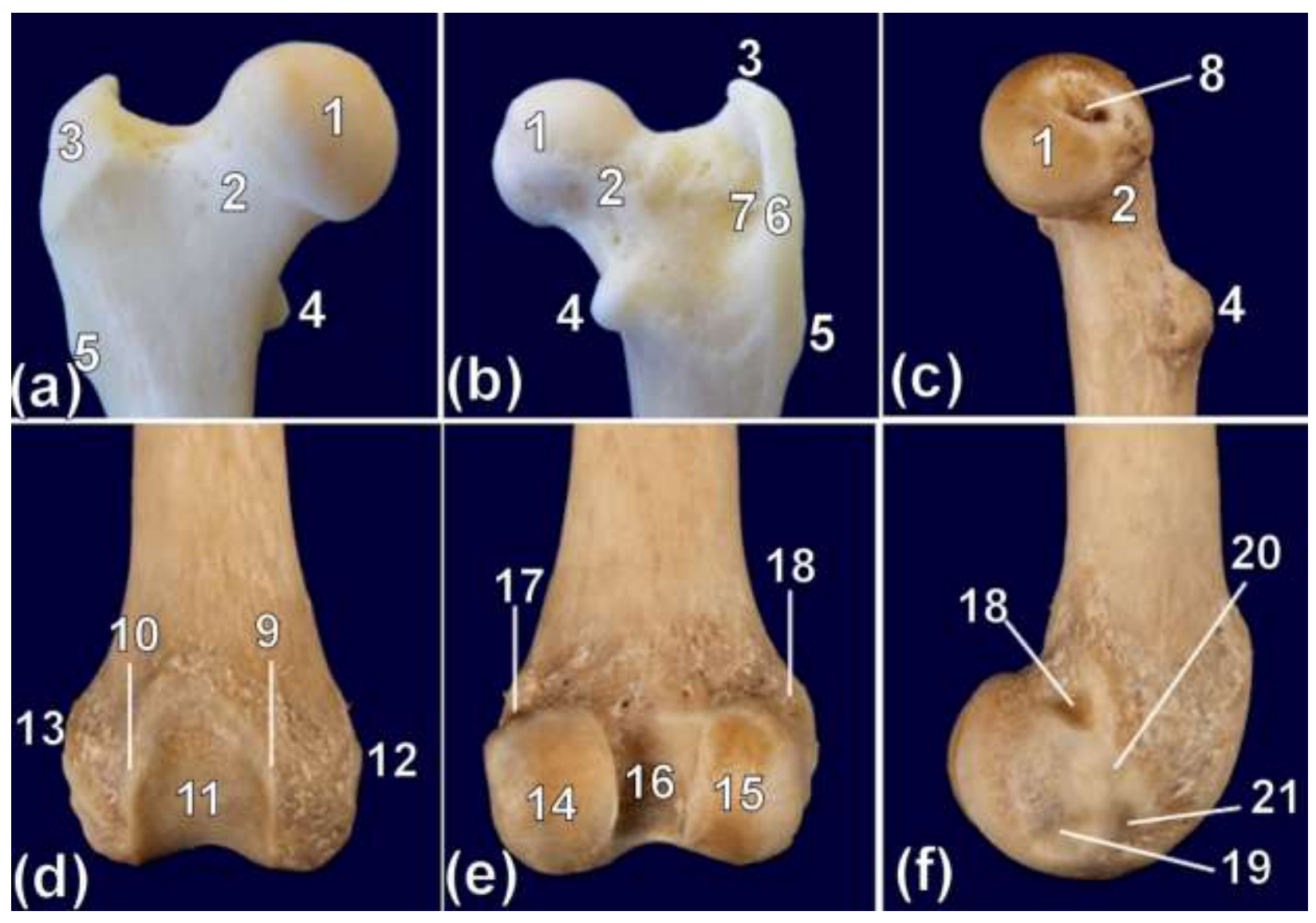

Fig. 3. Cranial (a), caudal (b) and medial (c) views of the proximal, and cranial (d), caudal (e) and lateral (f) views of the distal bone specimens of the femur of a 3.5-year-old (a, b) and a 14.3-year-old (c, d, e, f) male red pandas. (1) head, (2) neck, (3) major trochanter, (4) minor trochanter, (5) third trochanter, (6) edge of the major trochanter, (7) trochanteric fossa, (8) fovea capitis femoris, (9) medial ridge of trochlea, (10) lateral ridge of trochlea, (11) trochlea, (12) medial epicondyle, (13) lateral epicondyle, (14) medial condyle, (15) lateral condyle, (16) inter-condylar fossa, (17) fossa for the insertion of the medial head of the m. gastrocnemius, (18) fossa for the insertion of the lateral head of the m. gastrocnemius, (19) popliteal fossa, (20) fossa for the lateral collateral ligament, (21) extensor fossa.

\section{Femur}

\section{Gross osteology}

Generally the femur was straight and slender. The head of the femur was spherical in shape and directed medially (Fig. 3a-c). It had a very prominent fovea capitis femoris 
(Fig. 3c). The major trochanter did not extend proximal to the head of the femur (Fig. 3ac). The medially directed minor trochanter was prominent (Fig. 3a-c) and not connected to the major trochanter by an inter-trochanteric crest (Fig. 3b). The third trochanter was poorly developed and presented with a rough surface (Fig. 3a,b)

The medial and lateral ridges of the trochlea of the femur ran parallel to each other with the lateral ridge extending more proximal than its medial counterpart (Fig. 3d). The trochlea of the femur was wide and shallow (Fig. 3d). Medial and lateral to the proximal border of the caudal surface of the medial and lateral condyles, respectively, the distal part of the femur had fossae for the origin of the medial and lateral heads, respectively, of the $\mathrm{m}$. gastrocnemius (Fig. 3e,f). The articular facet for the lateral sesamoid bone of the $\mathrm{m}$. gastrocnemius, further indicated as the lateral fabella, was visualised on bone specimens of two animals. The lateral surface of the distal part of the femur had three fossae for the lateral collateral ligament and origin of the popliteus and extensor digitorum longus muscles (Fig. 3f). The popliteal fossa was more pronounced than the extensor fossa (Fig. 3f). The medial surface of the distal part of the femur had a fossa for the medial collateral ligament. The fossa for the medial collateral ligament was more prominent than its lateral counterpart.

\section{Radiography}

On the $\mathrm{CrCd}$ view, the major trochanter did not extend proximal to the head of the femur and the minor trochanter was prominent (Fig. 1c). The edge of the major trochanter ran distally (Fig. 1c). On the ML view, the shaft of the femur was long and slender (Fig. 4a). The cranial and caudal diaphyseal cortices of the femur were of almost uniform thickness (Fig. 4a). Radiographic measurements are shown in Table 2. 


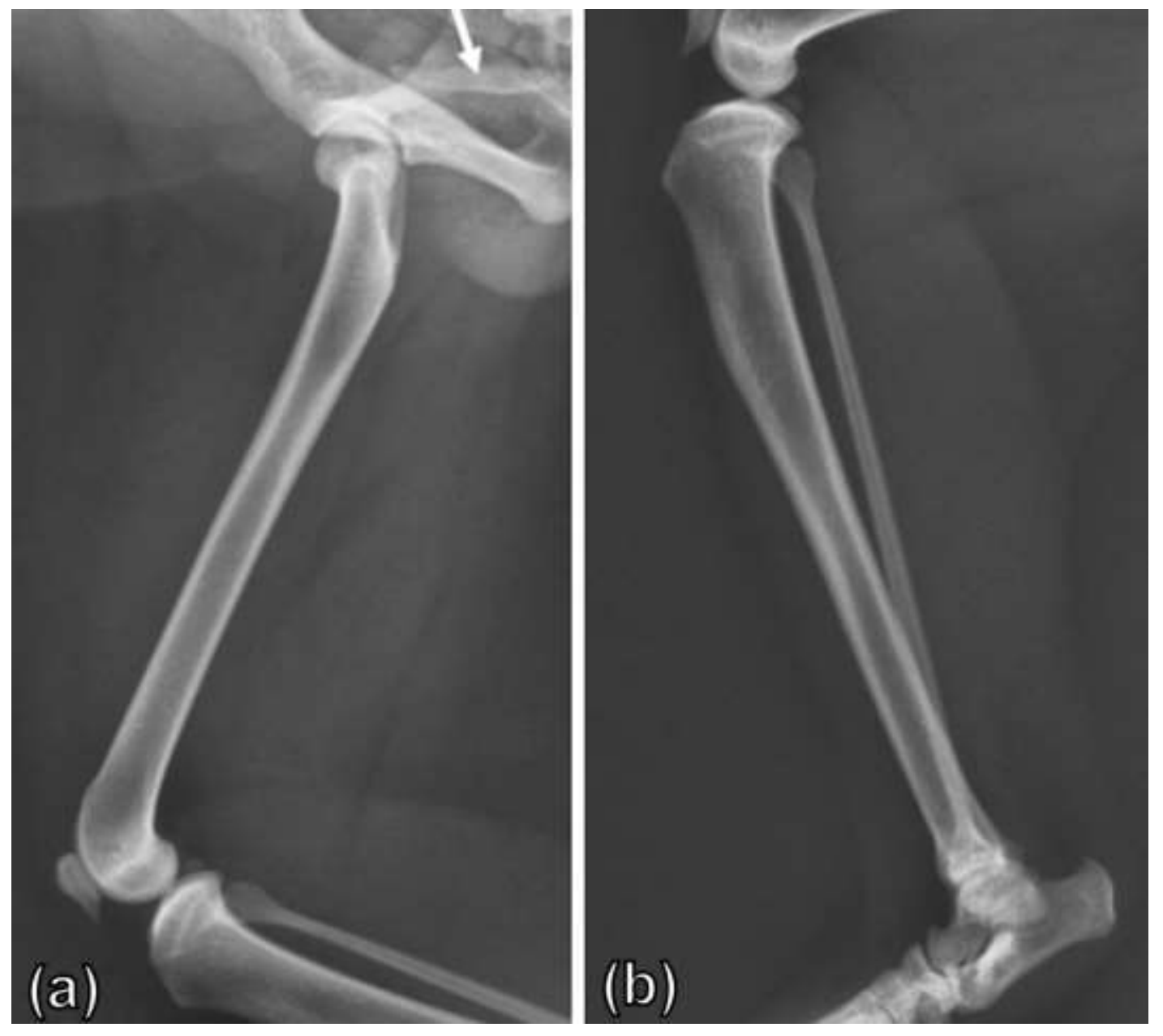

Fig. 4. Mediolateral radiograph of the right femur (a), tibia and fibula (b) of a 1.4-year-old male red panda. (a) The femur appears slender with very symmetric thicknesses of the cranial and caudal diaphyseal cortices. The os penis is indicated by the white arrow. (b) Note the variation in thickness of the cranial and caudal diaphyseal cortices of the tibia and an almost uniform thickness of the cranial and caudal diaphyseal cortices of the fibula. The body of the tibia is curved cranially. Note also the bulbous appearance of the proximal and distal parts of the fibula.

\section{Stifle}

Gross osteology, radiography and CT

The patella was similar to that seen in feline species with a pointed and elongated apex (Figs 5 and 6a). The lateral fabella was seen radiographically in 5 of 7 animals (Fig. 5), 


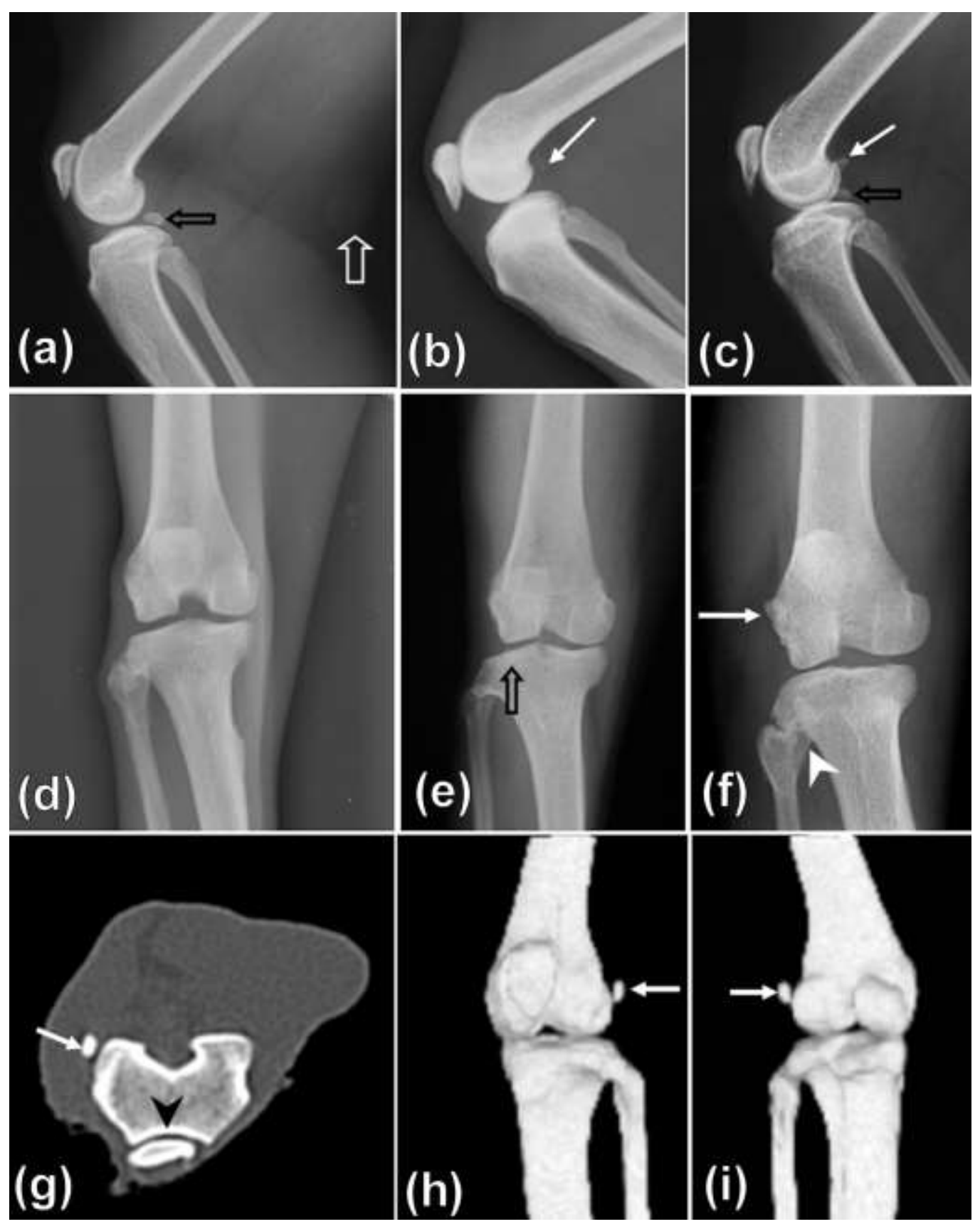

Fig. 5. Mediolateral (a, b, c) and $\mathrm{CrCd}(\mathrm{d}, \mathrm{e}, \mathrm{f})$ radiographs of the right stifle joint of 2.5-year-old (a), 14.3year-old (b, d), 1.4-year-old (c, e) and 11.3-year-old (f) male (a, b, d, e, f) and female (c) red pandas. Computed tomographic images $(\mathrm{g}, \mathrm{h}, \mathrm{i})$ of the left stifle joint of a 14.3-year-old male red panda. Transverse slice $(\mathrm{g})$ displayed using a sharp (B80) kernel with a bone window (WW=2000; WL=400). Craniolateral (h) and caudomedial (i) views of the 3D volume-rendered computed tomography image of a left stifle joint (using a grey scale default). Note the elongated and pointed apex of the patella, large popliteal sesamoid bone (open black arrows), and low projection of the tibial tuberosity and inter-condylar 
eminence. Note also the visualization of the lateral fabella (white arrows) and popliteal lymph node (open white arrow). Note the lack of visualization of the medial fabella in all animals. The popliteal sesamoid bone is poorly visualized and superimposed on the lateral condyle of the tibia (e). Note also the ankylosis of the tibiofibular joint (white arrow head) (f) and the shallow trochlea of the femur (black arrow head) $(\mathrm{g})$.
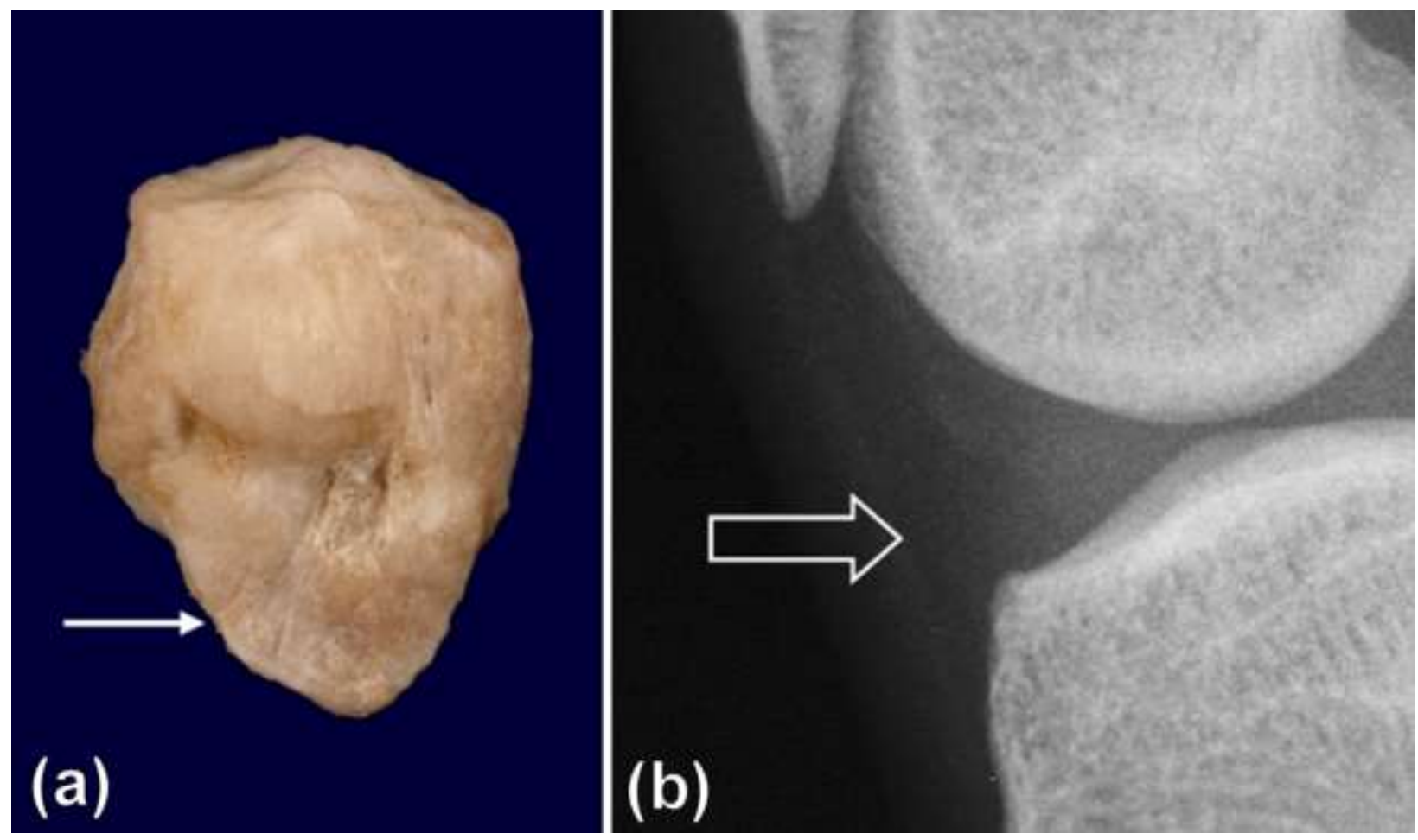

Fig. 6. Dorsal view (a) of a bone specimen of the patella of a 14.3-year-old male red panda. Close-up ML radiograph (b) of the right stifle joint of a 2.5-year-old male red panda. (a) Note the elongated and pointed apex of the patella (white arrow). (b) Note also the visualization of the infrapatellar fat pad (open white arrow).

whereas a medial fabella was not visualised radiographically in any animal (Fig. 5). The popliteal sesamoid bone was seen radiographically in 5 of 7 animals (Fig. 5). The trochlea of the femur was wide and shallow (Fig. $5 \mathrm{~g}$ ).

On the ML view, the caudal surface of the apex of the patella sloped from caudoproximal to craniodistal (Fig. 5a-c). The popliteal lymph node (Fig. 5a) and infrapatellar fat pad (Fig. 6b) were seen. The lateral fabella was mostly seen as ovoid 
elongated craniocaudally (Fig. 5c) and rarely angular or rounded (Fig. 5b). The popliteal sesamoid bone was large and located just proximal to the condyles of the tibia and caudal to the inter-condylar eminence (Fig. 5a,c). It was ovoid elongated craniocaudally (Fig. 5a,c). The inter-condylar eminence and tibial tuberosity were not prominent (Fig. $5 a-c)$.

On the $\mathrm{CrCd}$ view, the proximal border of the patella was fairly straight (Fig. $5 d-f)$. The lateral fabella (Fig. 5f) and popliteal sesamoid bone (Fig. 5f) were ovoid. The popliteal sesamoid bone was mostly seen superimposed on the lateral condyle of the tibia (Fig. 5e) and rarely on the lateral joint space. The inter-condylar eminence was not prominent (Fig. 5-f). Radiographic measurements are shown in Table 2.

\section{Tibia and fibula}

\section{Gross osteology}

The larger tibia and smaller fibula were unfused bones. The proximal articular surface of the tibia was located slightly caudally (Fig. 7b). The lateral condyle was slightly convex, whereas the medial condyle was concave. The inter-condylar eminence and tibial tuberosity were not prominent (Fig. 7a,b). The flat and ovoid caudolaterally located facies articularis fibularis of the tibia was prominent (Fig. 7b). The cochlea grooves were shallow, and the intermediate ridge was poorly defined. The medial malleolus was wide craniocaudally and presented with prominent grooves for the digital flexors. The facies articularis malleoli medialis sloped from lateral to medial (Fig. 7c,d). The lateral surface of the distal part of the tibia had a distinct facet, the fibular notch, for articulation with the distal part of the fibula (Fig. 7c,d). The head and lateral malleolus of the fibula were well developed (Fig. 7e,f). The head of the fibula had a distinct proximomedial facet (facies 


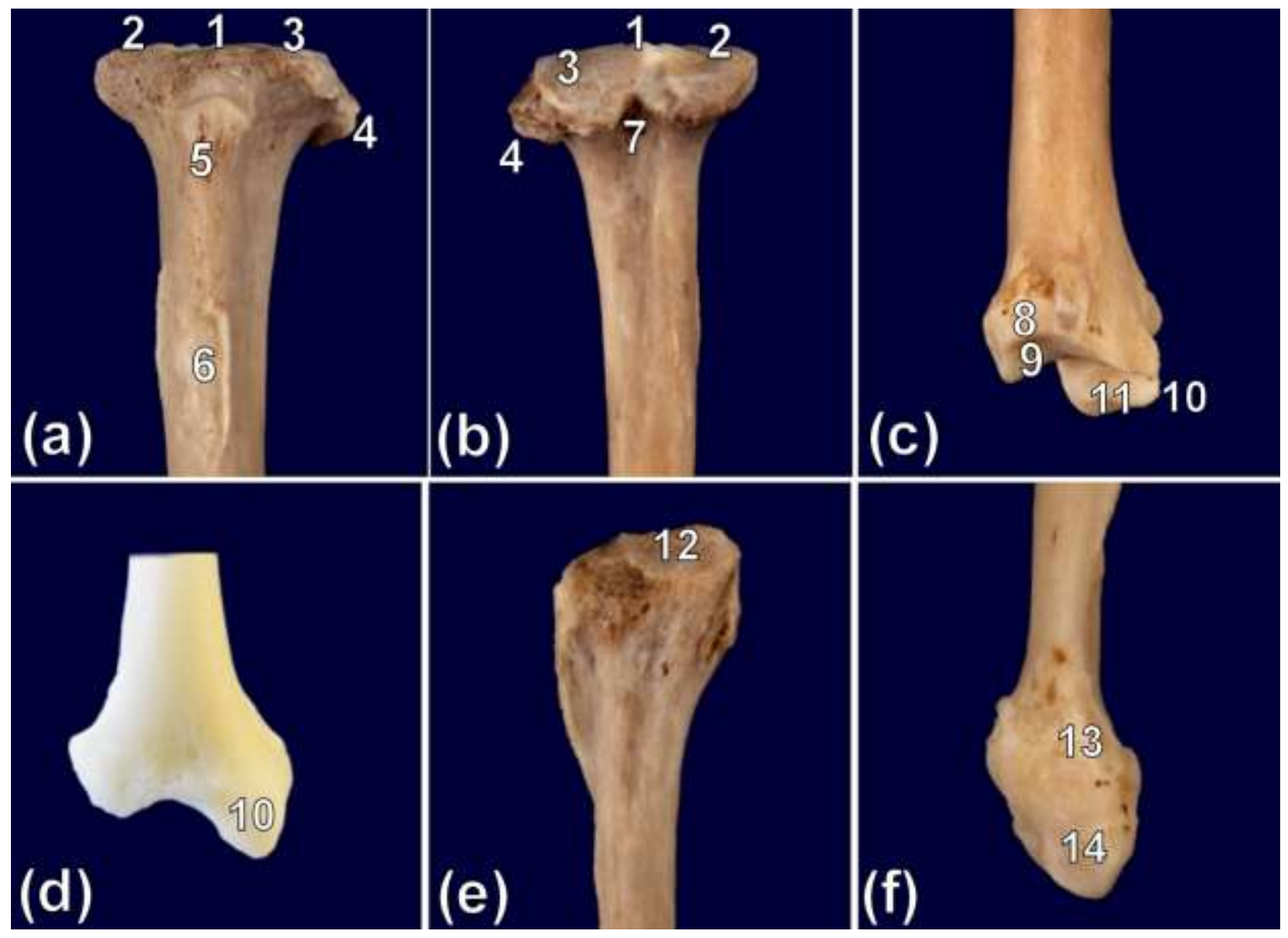

Fig. 7. Cranial (a, d), caudal (b) and caudolateral (c) views of the proximal and distal bone specimens of the tibia of an 11.3-year-old (a, b, c) and a 3.5-year-old (d) male red pandas. (e, f) Medial view of the proximal (e) and distal (f) bone specimen of the fibula of a 11.3-years-old male red panda. (1) Intercondylar eminence, (2) medial condyle, (3) lateral condyle, (4) facies articularis fibularis, (5) tibial tuberosity, (6) area for attachment of sartorius, gracilis and semitendinosus muscles, (7) popliteal notch, (8) fibular notch, (9) cochlea tibiae, (10) medial malleolus, (11) facies articularis malleoli medialis, (12) facies articularis capitis fibularis, (13) articular surface for the fibular notch of the tibia, (14) facies articularis malleoli lateralis.

articularis capitis fibularis) for articulation with the proximal part of the tibia (Fig. 7e). The body of the fibula was less compressed transversely. The lateral malleolus had two distinct medially located articular facets, which were proximal and distal to each other 
(Fig. 7f). The distal articular facet (facies articularis malleoli lateralis) sloped from medial to lateral and was fairly flattened. The proximal articular facet, which articulated with the fibular notch of the tibia, was slightly concave (Fig. 7f).

\section{Radiography}

On the ML view, the body of the tibia was fairly straight with a thicker cranial cortex than the caudal cortex (Fig. 4b). The head and lateral malleolus of the fibula were bulbous (Fig. 4b). The body of the fibula appeared with almost uniform thickness (Fig. 4b). The cranial and caudal diaphyseal cortices of the fibula were of uniform thickness (Fig. 4b). The inter-osseous space was broad and ran the entire length of the crus (Figs $4 b, 5$ and 8). Radiographic measurements are shown in Table 2.

\section{Tarsus}

Gross osteology, radiography and CT

The tarsus consisted of seven tarsal bones namely calcaneus, talus, central tarsal bone as well as first ( $\mathrm{T} \mathrm{I}$ ) through fourth (T IV) tarsal bones (Fig. 8a). The calcaneus was the largest and longest bone located laterally in the proximal row of the tarsus (Fig. 8). The proximal half of the bone was narrow transversely, whereas the distal half was wide transversely with two processes. The medial process (sustentaculum tali) was wide, whereas the lateral process was pointed (Fig. 8a). The dorsal surface of the calcaneus had three articular surfaces: the proximal, middle and distal articular surfaces. The proximal articular surface was the largest with a less convex articular surface, which articulated with the plantarolaterally located proximal articular surface of the talus. The dorsomedially located middle articular surface was slightly concave and articulated with 


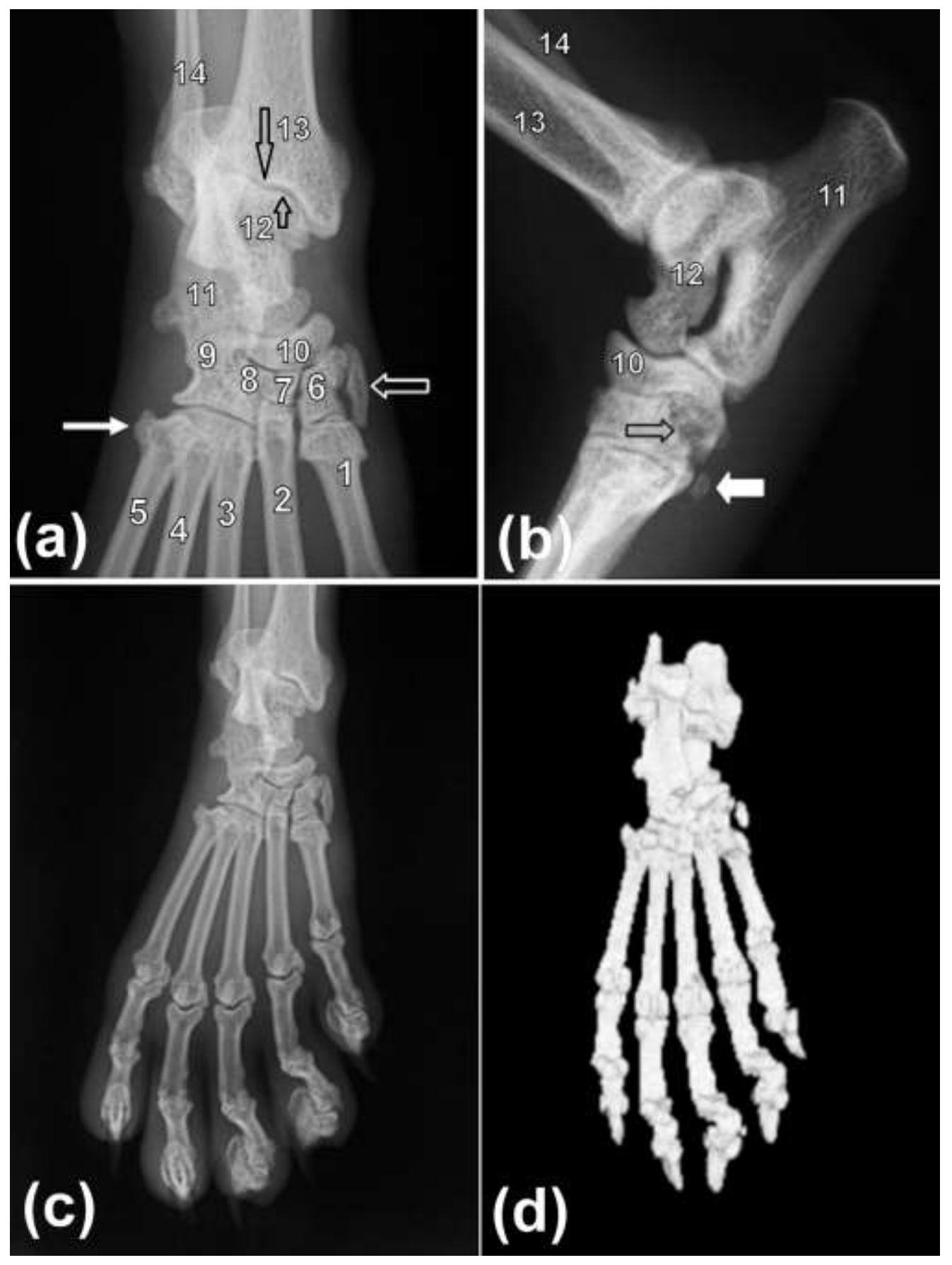

Fig. 8. Plantarodorsal (a, c) and mediolateral (b) radiographs of the right tarsus (a, b) and pes (c) of a 2.5year-old male (a, c) and a 1.4-year-old female (b) red panda. Plantar view (d) of the 3D volume-rendered computed tomography image of the left pes of a 14.3-year-old male red panda. (a) Note the low projection of the intermediate (long black open arrow) and medial (short black open arrow) ridges of the distal articular surface of the tibia and of the trochlea of the talus, respectively. Note also the laterally directed lateral process of the base of the metatarsal (MT) $\vee$ (white arrow) and the presence of a tarsal sesamoid 
bone (white open arrow). Note the sloping of the facies articularis malleoli medialis from lateral to medial. (b) Note also the presence of a small MT sesamoid bone on the plantar aspect of the bases of the MT bones (thick white arrow) and the wide talocalcaneal joint space. The focal radiolucent region seen just distal to the calcaneus (open black arrow) is normal and should not be confused with pathology. (c, d) Note the presence of paired axial and abaxial proximal sesamoid bones on each metatarsophalangeal joint and widely spaced MT bones and digits. (1) MT I, (2) MT II, (3) MT III, (4) MT IV, (5) MT V, (6) T I, (7) T II, (8) T III, (9) T IV, (10) central tarsal bone, (11) calcaneus, (12) talus, (13) tibia, (14) fibula.

the plantaromedially located middle articular surface of the talus, whilst the smallest distal articular surface was elongated mediolaterally.

The talus was the second largest bone and was located on the medial side of the proximal row of the tarsus (Fig. 8). The trochlea of the talus was shallow with a prominent lateral ridge and an almost flattened medial ridge (Fig. 8a). The head of the talus was elongated transversely. The plantar surface of the talus had three articular surfaces: the proximal, middle and distal ones. The plantarolaterally located proximal articular surface was the largest with a slightly concave articular surface. The plantaromedially located middle articular surface had a slightly convex articular surface. The distal articular surface was the smallest and articulated with the distal articular surface on the dorsal surface of the calcaneus.

The central tarsal bone was the only bone located in the middle row (Fig. 8). It was elongated transversely and shortened longitudinally (Fig. 8a). The bone had prominent proximal and distal articular surfaces, which were concave and convex, respectively (Fig. 8a,b). The T IV was the most lateral bone, whereas the $\mathrm{T}$ I was the most medial bone in the distal row (Fig. 8a). The T I had a proximomedial articular facet for articulation with the tarsal sesamoid bone on the medial aspect of the distal row of 
the tarsus (Fig. 8a). The T II was the shortest bone longitudinally in the distal row. In all animals, a large tarsal sesamoid bone was seen on the medial aspect of T I on the PD view of the pes (Fig. 8a). A distinct articulation was seen between the tarsal sesamoid bone and T I (Fig. 8a,c,d). The shape of the tarsal sesamoid bone varied. It was mainly seen as comma-shaped elongated longitudinally and rarely ovoid or angular. On the ML view, a focal radiolucent region was seen just distal to the calcaneus (Fig. 8b). On the PD view, the facies articularis malleoli medialis sloped from lateral to medial (Fig. 8a). The radiographic measurements of the tarsal sesamoid bone are shown in Table 2.

\section{Metatarsus and digits}

Gross osteology, radiography and CT

Five MT bones and digits were seen. The MT bones and digits were widely spaced (Fig. $8 \mathrm{c}, \mathrm{d})$. The lateral process of the base of the MT V was directed laterally (Fig. 8a,c,d). The heads of the MT bones were wider than the shafts. Digits II to V had proximal (P1), middle (P2) and distal (P3) phalanges, whereas digit I had only phalanges P1 and P3 (Fig. 8c,d). The distal articular surface of P2 was fairly symmetrical. The P3 had a concave proximal articular surface, which was parallel to the horizontal plane. The unguicular crests were prominent (Fig. 8c). On the ML view, a small semicircular metatarsal sesamoid bone was seen on the plantar aspect of the pes caudal to the bases of the MT bones (Fig. 8b and Table 2). Paired (axial and abaxial) proximal sesamoid bones were seen on the plantar side of each metatarsophalangeal joint (Fig. 8c,d). Radiographic measurements are shown in Table 3. 
Table 3. Radiographic measurement, not compensated for magnification, of length of short bones of the right hind limb in captive red pandas

\begin{tabular}{llll}
\hline Bone & Number of animals & Mean \pm SD & Range $(\mathrm{cm})$ \\
& & $(\mathrm{cm})$ & \\
\hline MT I & 6 & $2.20 \pm 0.13$ & $2.00-2.30$ \\
MT II & 6 & $3.08 \pm 0.17$ & $2.80-3.30$ \\
MT III & 6 & $3.43 \pm 0.18$ & $3.10-3.60$ \\
MT IV & 6 & $3.62 \pm 0.19$ & $3.30-3.80$ \\
MT V & 6 & $3.37 \pm 0.20$ & $3.00-3.50$ \\
P1 of digit I & 6 & $1.42 \pm 0.08$ & $1.30-1.50$ \\
P1 of digit II & 6 & $1.68 \pm 0.12$ & $1.50-1.80$ \\
P1 of digit III & 6 & $1.93 \pm 0.08$ & $1.80-2.00$ \\
P1 of digit IV & 6 & $1.87 \pm 0.10$ & $1.70-2.00$ \\
P1 of digit V & 6 & $1.47 \pm 0.08$ & $1.30-1.50$ \\
P2 of digit II & 6 & $1.21 \pm 0.08$ & $1.10-1.30$ \\
P2 of digit III & 6 & $1.25 \pm 0.06$ & $1.20-1.30$ \\
P2 of digit IV & 6 & $1.27 \pm 0.10$ & $1.10-1.40$ \\
P2 of digit V & 6 & $1.01 \pm 0.13$ & $0.80-1.20$ \\
\hline
\end{tabular}

\section{Other findings}

Fusion of the dorsal surface of the wing of the sacrum and ilium was seen in a 14.3year-old-animal (Fig. 1a). Unilateral lumbarisation of the first sacral vertebra was seen in a 10.3-year-old animal (Fig. 9a). Osteoarthrosis (OA) of the hip joint was seen bilaterally in a 11.3-year-old animal and was characterized by osteophyte formation around the neck of the femur and in the trochanteric fossa (Fig. 9d,e). Osteoarthrosis of the stifle joint was seen in a 9.6-year-old animal and was characterized by the presence of 
enthesiophytes on the apex of the patella and tibial tuberosity (Fig. 9b,c). In the tarsus OA was seen in three animals older than 9-years and was characterized by poor delineation of the tarsal bones and bases of the MT bones (Fig. 9c). Fusion of the tibiofibular joint was seen in two animals above 10-years (Figs $5 f$ and 9f).

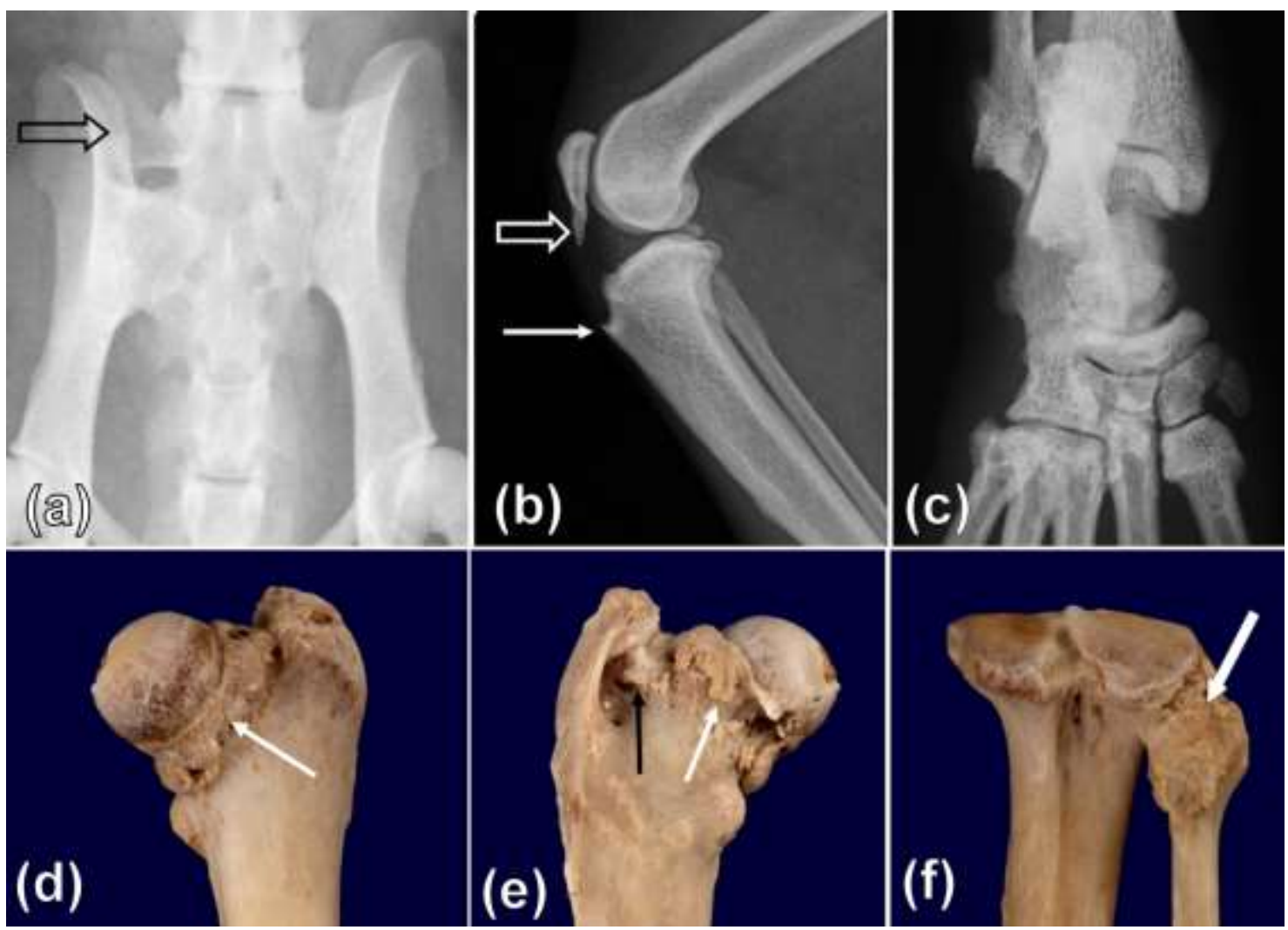

Fig. 9. Close-up VD radiograph of the pelvis of a 10.3-year-old male red panda (a), mediolateral radiograph of the right stifle joint of a 9.6-year-old female red panda (b) and PD radiograph of the right tarsus of a 14.3-year-old male red panda. Cranial $(d)$ and caudal $(e, f)$ views of bone specimens of the proximal femur (d, e) and fibula (f) of a 11.3-year-old male red panda. (a) Note the unilateral lumbarization of the first sacral vertebra (open black arrow). (b) Note also the presence of enthesiophytes on the apex of the patella (open white arrow) and tibial tuberosity (white arrow). (c) Note the poor delineation of the tarsal bones and bases of the metatarsal bones when compared to Fig. 8a. (d, e) Note also the presence of osteophytes at the femoral neck (white arrows) and in the trochanteric fossa (black arrow). (f) Note the ankylosis of the tibiofibular joint (thick white arrow). 


\section{Discussion}

The red panda is a quadrupedal arboreal animal spending most of its time in trees (Srivastan et al., 2009). Movement in trees is facilitated by a high degree of flexibility of the pelvic and pectoral girdles and limb joints (Roberts and Gittleman, 1984).

The more medially directed minor trochanter permits greater rotation of the femur and is associated with climbing ability (Taylor, 1976). The poorly defined intermediate ridge at the distal articular surface of the tibia and the less pronounced medial ridge of the trochlea of the talus facilitate supination of the foot (Taylor, 1976). Prominent medial and intermediate ridges of the talus and distal articular surface of the tibia, respectively, restrict the movement of the tibiotalar joint in flexion and extension for terrestrial locomotion (Taylor, 1976; Fleagle and Simons, 1995). Slightly concave and convex proximal and middle articular surfaces, respectively, on the plantar aspect of the talus, which articulate with the calcaneus, allow for greater mobility of the talocalcaneal joint (Taylor, 1976). The laterally directed lateral process of the base of the MT V most likely aids in the abduction of the foot and/or MT V as was speculated in climbing African Viverridae (Taylor, 1976). In a study which involved African Viveridae (Carnivora), the lateral process of the base of the MT V was found to be directed laterally in climbing species, whereas in terrestrial species, it was directed proximally (Taylor, 1976). The latter orientation permits very limited extension and flexion (Taylor, 1976), probably as an adaptation to a terrestrial environment. The widely spaced MT bones provide a large space for the effectiveness of the muscles, which are responsible for small movements of the digits (Taylor, 1974). 
Unlike in the red panda, the medial ridge of the talus and intermediate ridge at the distal articular surface of the tibia are more prominent in the coati (Nasua nasua) (Martins et al., 2013). The coati lives both on the ground and in trees (McClearn, 1992). Although it can descend or ascend small trees and vines, it is primarily a terrestrial animal (Gompper and Decker, 1998). The coati is not a graceful climber (McClearn, 1992) and is unable to fully reverse its hind foot during descending (McClearn, 1992; Gompper and Decker, 1998) as does the red panda.

The wide ventromedial surface of the ilium and the medially directed minor trochanter observed in this species are associated with climbing ability. The wide ventromedial surface of the ilium provides a large area for the origin of the $\mathrm{m}$. iliacus, which inserts on the minor trochanter (Fisher et al., 2008). The wide ventromedial surface of the ilium most likely indicates that the $\mathrm{m}$. iliopsoas is powerful in this species.

The caudal ramus of the pubis provides the origin of the various adductors of the hind limb (Fisher et al., 2008). This wide ramus of the pubis observed in this species is almost similar to the coati (Martins et al., 2013) and most likely indicates the strength of these muscles in adduction of the hind limb. It is most likely that these muscles, together with the ability to supinate the foot, play a greater role in the gripping action of the feet.

The shallow trochlea of the femur, the short widely spaced MT bones and the presence of a well-developed MT I observed in the red panda are adaptations to arboreal locomotion. The trochlea of the femur is well-developed and surrounded by high ridges in cursorial animals and those that rely on rapid or powerful knee extension (Taylor, 1976; Fleagle and Simons, 1995). Further, in cursorial species, the MT bones are long and close to each other with the MT I being reduced or absent (Taylor, 1976). In the coati (Martins et al., 2013), the MT bones are widely spaced and MT I is well- 
developed similar to the red panda. This is because both the red panda and coati have the ability to climb on trees.

A distinct articular facet between the distal parts of the tibia and fibula observed in this species is similar to the coati (Martins et al., 2013) and is associated with the plantigrade posture (Taylor, 1976). Plantigrade posture has been reported in the coati (Gompper and Decker, 1998). The fairly symmetrical distal articular surface of P2 and the parallel orientation to the horizontal plane of the proximal articular surface of P3 observed in this species indicate that the claws are slightly (Taylor, 1974) to nonretractile (Gonyea and Ashworth, 1975).

The large ovoid popliteal sesamoid bone observed in this study appears similar to that of domestic cats (McCarthy and Wood, 1989). The absence of visualization of the popliteal sesamoid bone on radiographic examination in some of the animals in this study is similar to a reported study in domestic cats (Arnbjerg and Heje, 1993). The medial fabella may not be visualized radiographically in domestic cats (Arnbjerg and Heje, 1993), which is also similar to our findings. The tarsal sesamoid bone observed in the red panda provides the origin for the medial belly of the $\mathrm{Mm}$. flexores digiti I profundi breves (Fisher et al., 2008). We suggest the sesamoid bone to be named as the medial sesamoid bone for the Mm. flexores digiti I profundi breves. The tarsal sesamoid bone is also present in the coati (Martins et al., 2013). The focal radiolucent region seen just distal to the calcaneus in all animals on the $M L$ radiographic view is a relative radiolucency as a result of less bone in this area (3D effect of bones) and should not be misinterpreted as osteolysis due to pathological conditions.

Osteoarthrosis of the hip joint observed in this study was characterized mainly by remodelling of the neck of the femur similar to dogs but different from domestic cats 
(Biery, 2006; Lascelles, 2010). In the latter species, it is characterized mainly by extensive remodelling and proliferative changes involving the craniodorsal acetabular margin and minimal remodeling of the neck of the femur (Biery, 2006; Lascelles, 2010). The normal pointed appearance of the patella in this species could make the decision of whether early enthesiophyte formation is present very difficult.

Radiographic measurements obtained in this study will be slightly increased due to magnification (Thrall, 2013). Object-film distance affects magnification more than focal spot-film distance. This is because the latter is usually kept constant. In clinical practice, some parts of the patient are always farther from the film, which causes magnification. Increasing object-film distance increases magnification (Thrall, 2013). It was decided not to compensate for magnification to provide more useful data for clinical use, where compensation for magnification is also not applied.

The morphology of the pelvis and hind limb of the red panda indicated flexibility of the pelvis and hind limb joints as an adaptation to arboreal quadrupedal behaviour. Further, this species possesses some unique anatomical features such as a tarsal sesamoid bone, a poorly defined intermediate ridge at the distal articular surface of the tibia, a laterally directed lateral process of the base of MT V, widely spaced MT bones and digits, and a well-developed MT I and digit I. Knowledge of the variations in the normal morphology of the pelvis and hind limb of different animal species is essential for successful identification of lesions and skeletons. 


\section{Acknowledgements}

The authors would like to thank the Organization for Women in Science for the Developing World (OWSD), Swedish International Development Cooperation Agency (SIDA), University of Pretoria, Johannesburg (JHB) and Bristol Zoos for supporting this study. The National Museums of Scotland for supplying the bone specimen. Dr. Georgia, Ms. Fania Mohlala, sisters and animal handlers of the JHB zoo and Onderstepoort Veterinary Academic Hospital for their assistance during anaesthesia, CT and radiographic examinations. Mrs. Charmaine Vermeulen of the University of Pretoria for photographic assistance and Mrs. Wilma Olivier of University of Pretoria for her assistance in administrative work.

\section{References}

de Araújo, F. A. P., N. F. Sesoko, S. C. Rahal, C. R. Teixeira, T. R. Müller, and M. R. F. Machado, 2013: Bone morphology of the hind limbs in two caviomorph rodents. Anat. Histol. Embryol. 42, 114-123.

Arnbjerg, J., and N. I. Heje, 1993: Fabellae and popliteal sesamoid bones in cats. J. Small Anim. Pract. 34, 95-98.

Biery, D. N., 2006: The hip joint and pelvis. In: BSAVA Manual of Canine and Feline Musculoskeletal Imaging (F. J. Barr and R. M. Kirberger, eds). Gloucester: UK. pp. 119-134.

Delclaux, M., C. Talavera, M. López, J. M. Sánchez, and M. I. García, 2002: Avascular necrosis of the femoral heads in a red panda (Ailurus fulgens fulgens): possible Legg-CalvePerthes disease. J. Zoo Wildl. Med. 33, 283-285. 
Eriksson, P., J. Zidar, D. White, J. Westander, and M. Andersson, 2010: Current husbandry of red pandas (Ailurus fulgens) in zoos. Zoo Biol. 29, 732-740.

Fisher, R. E., 2011: Red panda anatomy. In: Red panda Biology and Conservation of the First Panda (A. R. Glatston, ed.). London: Academic Press. pp. 89-100.

Fisher, R. E., B. Adrian, C. Elrod, and M. Hicks, 2008: The phylogeny of the red panda (Ailurus fulgens): evidence from the hind limb. J. Anat. 213, 607-628.

Fisher, R. E., B. Adrian, M. Barton, J. Holmgren, and S. Y. Tang, 2009: The phylogeny of the red panda (Ailurus fulgens): evidence from the forelimb. J. Anat. 215, 611-635.

Fleagle, J. G., and E. L. Simons, 1995: Limb skeleton and locomotor adaptations of Apidium phiomense, an Oligocene Anthropoid from Egypt. Am. J. Phys. Anthropol. 97, 235-289.

Gompper, M. E., and D. M. Decker, 1998: Nasua nasua. Mamm. Species. 580, 1-9.

Gonyea, W., and R. Ashworth, 1975: The form and function of retractile claws in the felidae and other representative carnivorans. J. Morph. 145, 229-238.

Groves, C., 2011: The taxonomy and phylogeny of Ailurus. In: Red panda Biology and Conservation of the First Panda (A. R. Glatston, ed.). London: Academic Press. pp. 101-124.

Heath, T., and J. Platnick, 2008: Ailurus fulgens animal diversity web. Available at: http://animaldiversity.ummz.umich.edu/accounts/Ailurus_fulgens (accessed on 4 April 2013).

IUCN, 2014: IUCN Red List of Threatened Species. Version 2014.2. Available at: http://www.iucnredlist.org (accessed on 13 August 2014).

Kinns, J., R. Malinowski, F. McEvoy, T. Schwarz, and A. Zwingenberger, 2011: Special software applications in veterinary computed tomography. In: Veterinary Computed Tomography ( $\mathrm{T}$. Schwarz and J. Saunders, eds). UK: John Wiley \& Sons Ltd. pp. 67-74.

Lascelles, B. D. X., 2010: Feline degenerative joint disease. Vet. Surg. 39, 2-13. 
Lynch, M., H. McCracken, and R. Slocombe, 2002: Hyperostotic bone disease in red pandas (Ailurus fulgens). J. Zoo Wildl. Med. 33, 263-271.

Martins, G. S., E. R. Lopes, I. I. G. Taques, C. Y. Correia, Y. S. Meireles, N. C. M. R. Turbino, L. D. Guimarães, and P. B. Néspoli, 2013: Radiographic morphology of the skeleton, thorax and abdomen of coati (Nasua nasua Linnaeus, 1766). Pesq. Vet. Bras. 33, 1137-1143.

McCarthy, P. H., and A. K. W. Wood, 1989: Anatomical and radiological observations of the sesamoid bone of the popliteus muscle in the adult dog and cat. Anat. Histol. Embryol. $18,58-65$.

McClearn, D., 1992: Locomotion, posture and feeding behaviour of kinkajous, coatis and raccoons. J. Mamm. 73, 245-261.

Nickel, R., A. Schummer, E. Seiferle, J. Frewein, H. Wilkens, and K. H. Wille, 1986: The Anatomy of the Domestic Animals Vol. 1. Berlin: Springer-Verlag.

O'Brien, T., 1978: Normal radiographic anatomy of the abdomen. In: Radiographic Diagnosis of Abdominal Disorders in the Dog and Cat. Philadelphia: W. B. Saunders Company. pp. $9-47$.

Ohlerth, S., and G. Scharf, 2007: Computed tomography in small animals-basic principles and state of the art applications. Vet. J. 173, 254-271.

Philippa, J., and E. Ramsay, 2011: Captive red panda medicine. In: Red panda Biology and Conservation of the First Panda (A. R. Glatston, ed.). London: Academic Press. pp. 271-285.

Pradhan, S., G. K. Saha, and J. A Khan, 2001: Ecology of the red panda Ailurus fulgens in the Singhalila National Park, Darjeeling, India. Biol. Cons. 98, 11-18.

Preece, B., 2011: Red panda pathology. In: Red panda Biology and Conservation of the First Panda (A. R. Glatston, ed.). London: Academic Press. pp. 287-302.

Roberts, M. S., and J. L. Gittleman, 1984: Ailurus fulgens. Mamm. Species. 222, 1-8. 
Samii, V., I. Gielen, E. Ludewig, W. M. Adams, I. Kiefer, H. vanBree, and J. Saunders, 2011: Joints. In: Veterinary Computed Tomography (T. Schwarz and J. Saunders, eds). UK: John Wiley \& Sons Ltd. pp. 387-419.

Silverman, S., and L. A. Tell, 2005: Radiology of Rodents, Rabbits and Ferrets, An Atlas of Normal Anatomy and Positioning. St. Louis: Elsevier Saunders.

Srivastav, A., P. Nigam, D. Chakraborty, and A. K. Nayak, 2009: National Studbook of Red pandas (Ailurus fulgens). New Delhi: Wildlife Institute of India, Dehradun and Central Zoo Authority.

Taylor, M. E., 1974: The functional anatomy of the forelimb of some African Viverridae (Carnivora). J. Morph. 143, 307-335.

Taylor, M. E., 1976: The functional anatomy of the hindlimb of some African Viverridae (Carnivora). J. Morph. 148, 227-253.

Thrall, D. E., 2013: Introduction to radiographic interpretation. In: Textbook of Veterinary Diagnostic Radiology (D. E. Thrall, ed.), St. Louis: Saunders Elsevier. pp. 74-86.

Thrall, D. E., and I. D. Robertson, 2011: The pelvic limb. In: Atlas of Normal Radiographic Anatomy and Anatomic Variants in the Dog and Cat (D. E. Thrall and I. D. Robertson, eds). St. Louis: Elsevier Saunder. pp. 100-126.

Wei, F., and Z. Zhang, 2009: Family Ailuridae (red panda). In: Handbook of the Mammals of the World Vol. 1 Carnivores (D. E. Wilson and R. A. Mittermeier, eds). Barcelona: Lynx Edicions. pp. 498-503. 